\title{
Exosomes and Friends: A General Overview on Reproduction
}

\author{
Faranak Mohammadpour Lashkari ${ }^{1}$, Anahita Mohseni Meybodi ${ }^{2}$ and Marjan Sabbaghian ${ }^{1 *}$ \\ ${ }^{1}$ Department of Andrology, Reproductive Biomedicine Research Center, Royan Institute for Reproductive Biomedicine, ACECR, Tehran , Iran. \\ ${ }^{2}$ Department of Genetics, Reproductive Biomedicine Research Center, Royan Institute for Reproductive Biomedicine, ACECR, Tehran, Iran.
}

*Corresponding author: Marjan Sabbaghian, Department of Andrology, Reproductive Biomedicine Research Center, Royan Institute for Reproductive Biomedicine, ACECR, Tehran, Iran.

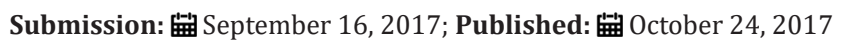

\section{Mini Review}

Nowadays, extracellular vesicles (EVs) as phospholipid bilayer enclosed vesicles are as popular as they have ever been. EVs are secreted by different cells and they target both tissues and/or cells in the body [1]. They play key roles in various aspects of biology, such as reproduction, as biomarkers of disease and as potent targets for therapeutic interposition [2]. Depending on their size, contents, biogenesis and function they have been classified as microvesicles, apoptotic bodies, ectosomes and exosomes [3].

About 50 years ago, extracellular membranous vesicles were introduced as cellular waste products. The exact definition of exosomes as a main member of EVs come from a study on the loss of transferrin during the maturation of reticulocytes into erythrocytes in 1983 [4]. Exosomes are nanovesicles about 40-100nm that are formed within cells by budding of late endosomes, called MVBs (multi-vesicular bodies) by releasing into the extracellular environment $[5,6]$. They are an enrich cargo of proteins and genetic materials. They are secreted by cells and found in most body fluids such as blood, plasma, saliva, breast milk, semen. Based on their cellular origin, they are contained specific protein markers namely tetra spanins (CD63, CD9 and CD81), heat-shock proteins (HSP70) and Rab family proteins $[3,7,8]$.

Exosomes are valuable vesicles since they show three important functions that attracted large numbers of scientists. First, they are able to make intercellular communication and transmit macromolecules between cells as a cellular vehicle. Second, they have been attributed roles in the spread of lipids, proteins, DNA and a variety of RNA species as key factors that impact on development of diseases. Third, they are proposed as useful drug vectors [9].

As mentioned before, exosomes are found in semen and also secreted by the male reproductive tract, especially by prostate and epididymis. They have been named prostasomes and epididymosomes, respectively. As striking features, they are differ in density, size and contents. Albeit their role in sperm physiology is not completely clear, but there are different studies on semen's exosomes and its roles on the fertility [1].
Prostasomes which are secreted from prostate gland measured 50-500 $\mathrm{nm}$ and they contain proteins, lipids and nucleic acids. As a transporter, human prostasomes are able to transfer at least 140 proteins that are important elements namely prostate-specific proteins (PSA and PAP), structural proteins, signal transduction, adenosine triphosphates and guanosine triphosphates [10]. Moreover, they have an important responsibility in controlling the exact time of capacitation and acrosome reaction. In particular, they prevent premature sperm capacitation and premature acrosome reaction. They are able to protect sperm cells from the female immune system and acidic environment on their way to the ovum [6].

Epididymosomes are secreted from epididymal epithelial cells that are one of the most interesting structures of the male reproductive tract. They are released via apocrine secretion and have been isolated from most of the species including bovine, mouse, hamster, rat as well as human $[1,6]$. They Contain adhesion molecules such as tetraspanins, integrins and other proteins. These proteins are involved in sperm maturation and fertilization, promote sperm motility, prevent premature acrosome reaction and capacitation and improve sperm penetration through the oocyte. Epididymosomes Fuse with the sperm cells to transfer proteins that contribute to the maturation of sperm cells [6].

In female reproduction system, there are bidirectional communication between granulosa cells and gametes that occur directly by a network of gap junctions or through autocrine, paracrine and endocrine signaling factors in the follicular fluid [11]. EVs specially exosomes are able to add an additional layer of control and transmission, since they carry miRNAs that are target key elements in pathways related to follicular growth and oocyte maturation (in mammals), such as transforming growth factor beta (TGFb), mitogen-activated protein kinase (MAPK), epidermal growth factor receptor (ErbB) pathways, wingless signaling pathway (WNT), ubiquitin mediated pathways and neurotrophin [12]. In most of the species, follicular fluid exosomes contain miRNAs which may contribute to either follicular growth and oocyte maturation [13]. 
According to new studies, EVs are much liked by researchers. It's certainly true that they are transferring important elements from cargo through the body per day. Whereas almost, their functional roles are not distinctly understood, as it mentioned before, they are able to contribute in oocyte and sperm maturation, embryo implantation, fertilization and polyspermy prevention. Most studies on the exosomes have been used animal models, albeit related human research is emerging. It seems that there is a critical need for further functional studies to report decisive experimental evidence for exosomes and their valuable cargo as intercellular communication mediator.

\section{References}

1. Sullivan R, Saez F (2013) Epididymosomes, prostasomes, and liposomes: their roles in mammalian male reproductive physiology. Reproduction 146(1): R21-35.

2. Gould SJ, Raposo G (2013) As we wait: coping with an imperfect nomenclature for extracellular vesicles. J Extracell Vesicles 2.

3. Bobrie A, Colombo M, Raposo G, Théry C (2011) Exosome secretion: molecular mechanisms and roles in immune responses. Traffic 12(12): 1659-1668.

4. Harding C, Heuser J, Stahl P (1983) Receptor-mediated endocytosis of transferrin and recycling of the transferrin receptor in rat reticulocytes. J Cell Biol 97(2): 329-339.

5. Laulagnier K, Motta C, Hamdi S, Roy S, Fauvelle F, et al. (2004) Mast cell- and dendritic cell-derived exosomes display a specific lipid composition and an unusual membrane organization. Biochem J 380(Pt 1): 161-171.

6. Machtinger R, Laurent LC, Baccarelli AA (2016) Extracellular vesicles: roles in gamete maturation, fertilization and embryo implantation. Hum Reprod Update 22(2): 182-193.

7. van der Pol E, Böing AN, Harrison P, Sturk A, Nieuwland R (2012) Classification, functions, and clinical relevance of extracellular vesicles. Pharmacol Rev 64(3): 676-705.

8. Properzi F, Logozzi M, Fais S (2013) Exosomes: the future of biomarkers in medicine. Biomark Med 7(5): 769-778.

9. Edgar JR (2016) Q\&A: What are exosomes, exactly? BMC Biol 14: 46.

10. Ronquist GK, Larsson A, Stavreus-Evers A, Ronquist G (2012) Prostasomes are heterogeneous regarding size and appearance but affiliated to one DNA-containing exosome family. Prostate 72(16): 17361745 .

11. Matzuk MM, Burns KH, Viveiros MM, Eppig JJ (2002) Intercellular communication in the mammalian ovary: oocytes carry the conversation. Science 296(5576): 2178-2180.

12. Santonocito M, Vento M, Guglielmino MR, Battaglia R, Wahlgren J, et al. (2014) Molecular characterization of exosomes and their microRNA cargo in human follicular fluid: bioinformatic analysis reveals that exosomal microRNAs control pathways involved in follicular maturation. Fertil Steril 102(6): 1751-61e1.

13. da Silveira JC, Veeramachaneni DN, Winger QA, Carnevale EM, Bouma GJ (2012) Cell-secreted vesicles in equine ovarian follicular fluid contain miRNAs and proteins: a possible new form of cell communication within the ovarian follicle. Biol Reprod 86(3): 71. 